\title{
Infra Red Radiation Detection using Passive Infrared Sensor
}

\author{
Shafiqul Abidin \\ HMR Institute of \\ Technology and Management, \\ Hamdipur, Delhi - 110036 (India)
}

\author{
Manu Ahuja \\ HMR Institute of \\ Technology and Management \\ Delhi - 110036 (India)
}

\begin{abstract}
This paper proposes a new approach for infrared object localization and tracking with passive infrared sensors (PIR). The hierarchical architecture visibility of Fresnel lens away is presented with modulated field of view (FOV). The FOVs of lens array in sensor node are modulated to achieve a single degree of freedom (DOF). The energy imbalance problem effectively solve with the PIR system. PIR based system saves power consumption and memory space. Passive infrared system detects the change in the radiation of warm blood generation and completely used to turn On the webcam and lighting system.
\end{abstract}

\section{Keywords}

Passive Infrared Sensors (PIR); Field of View (FOW); style; styling; Degree of Freedom (DOF)

\section{INTRODUCTION}

Infrared (IR) refers to an invisible electromagnetic radiant energy. The wavelength of IR is longer than visible light. The frequency of IR is extended from the normal red edge of the visible spectrum at 700 nanometers. The frequency varies from $430 \mathrm{THz}$ to $300 \mathrm{GHz}$ [1]. However, from experimental results it is found that human can see IR up to $1050 \mathrm{~nm}$. [2, 3, 4, 5]. At room temperature, large number of things emit thermal radiations are basically infra red in nature.

Astronomer Sir William Herschel revealed Infrared radiation in 1800. Sir William found invisible radiation in the spectrum with lesser energy if compared with red light [6]. Further, half of the emitted energy from the sun is reached on the earth in the form of infra red. These radiations play a vital role on Earth's climate. Infrared energy is emitted or absorbed by molecules when they change their rotational-vibration.

Passive infrared sensors to develop an electric signal in response to a change in the incident thermal radiation [7]. Every living body emits some low level radiations and the hotter the body, the more is emitted radiation. Commercial PIR sensors typically include two IR-sensitive elements with opposite polarization housed in a hermetically sealed metal with a window made of IR-transmissive material (typically coated silicon to protect the sensing element). When the sensor is idle, both slots detect the same amount of IR, the ambient amount radiated from the room or walls or outdoors $[8,9]$. The block diagram of passive infrared motion sensor is shown in Fig. 1.

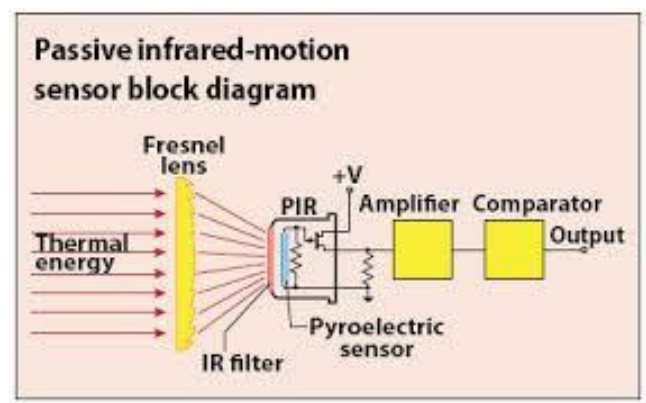

Fig. 1. PIR Motion Sensor

A warm body like a human passes by, it first intercepts one half of the PIR sensor which causes a positive differential change between the two halves. When the warm body leaves the sensing area, the reverse happens, whereby the sensor generates a negative differential change. These change pulses are what is detected. In order to shape the field of view (FOV) i.e. Field Of View of the sensor and the detector is equipped with lenses in front of it [10].

The lens used here is inexpensive and lightweight plastic materials with transmission characteristics suited for the desired wavelength range. To cover much larger area, detection lens is split up into multiple sections, each section of which is a Fresnel lens [11]. Fresnel lens condenses light. Providing a larger range of IR to the sensor it can span over several tens of degree width. Thus total configuration improves immunity to changes in background temperature, noise or humidity and causes a shorter settling time of the output after a body moved in or out the FOV. Along with piezoelectric sensor, a chip named Micro Power PIR Motion Detector circuit as shown in the Fig 3(a) and Fig 3 (b) which is used. The lens is just a piece of plastic, but that means that the detection area is just two rectangles. Usually we'd like to have a detection area that is much larger. we use a simple lens such as those found in a camera: they condenses a large area (such as a landscape) into a small one (on film or a CCD sensor). For reasons that will be apparent soon, we would like to make the PIR lenses small and thin and moldable from cheap plastic, even though it may add distortion [12]. For this reason the sensors are actually Fresnel lenses as shown in Fig 2.

A person enters through main gate, IR sensors gets activated and the Microcontroller indicates the correct direction, the buzzer beeps and an led glows Indicating the entry is authorised [13] .In this case no operation of motor and laser gun is involved. But a person enters the premises apart from the main gate then PIR sensors get activated and they provide a positive output to 4049IC. 4049 IC is an inverter IC which provide negative output to microcontroller and turns stepper motor ON which moves in the direction indicated and stops 
$[14,15]$.The laser gun turns $\mathrm{ON}$ which is indicated by the buzzer and red led blinks showing the person is shot with em wave in as shown in the Fig.4 [16].

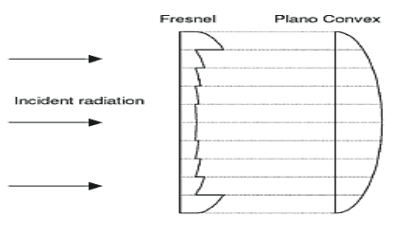

Fig. 2. Fresnel lenses

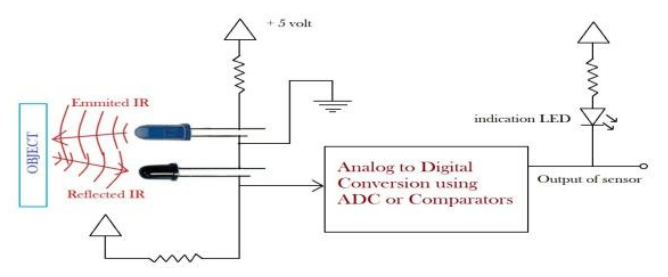

Fig. 3. PIR Sensor

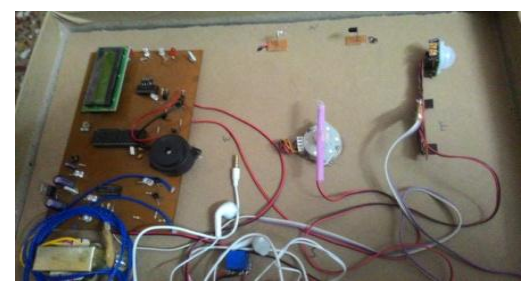

Fig. 3 (a). Micro Power PIR Motion Detector

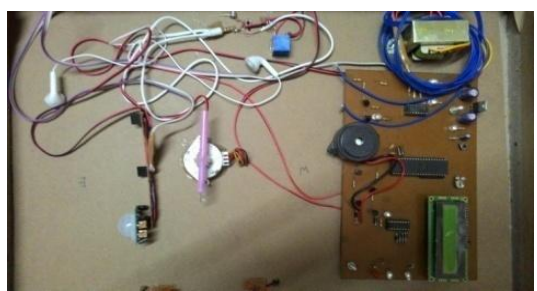

Fig. 3 (b). Micro Power PIR Motion Detector

Output Measuring Parameters of the Detectors are given in table 1.

Table 1. Output Measuring Parameters of the Detectors

\begin{tabular}{|l|l|l|l|l|l|l|}
\hline Ring & $\mathbf{S}$ & $\mathbf{S}$ & $\mathbf{S}$ & $\begin{array}{l}\text { Distanc } \\
\text { e from } \\
\text { the } \\
\text { Detecto } \\
\text { rs } \\
\text { Centre }\end{array}$ & $\begin{array}{l}\text { Estimat } \\
\text { ed } \\
\text { Distance }\end{array}$ & $\begin{array}{l}\text { Which } \\
\text { Detect } \\
\text { or } \\
\text { Sense } \\
\text { the } \\
\text { Object } \\
\text { S }\end{array}$ \\
\hline 1 & 0 & 0 & 0 & 0 & 0 & Nil \\
\hline 2 & 0 & 0 & 1 & $0-R 1$ & $\mathrm{R} 1 / 2$ & $\mathrm{~S} 3$ \\
\hline 3 & 0 & 1 & 0 & $\mathrm{R} 1-\mathrm{R} 2$ & $\mathrm{R} 1+\mathrm{R} 2 / 2$ & $\mathrm{~S} 2, \mathrm{~S} 3$ \\
\hline 4 & 0 & 1 & 1 & $\mathrm{R} 2-\mathrm{R} 3$ & $\mathrm{R} 2+\mathrm{R} 3 / 2$ & $\mathrm{~S} 2$ \\
\hline
\end{tabular}

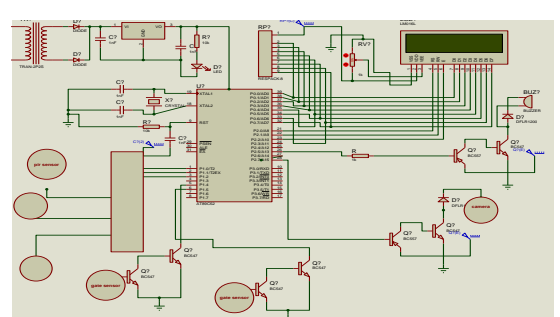

Fig. 4. Pyro Electricity to Detect a Human Body

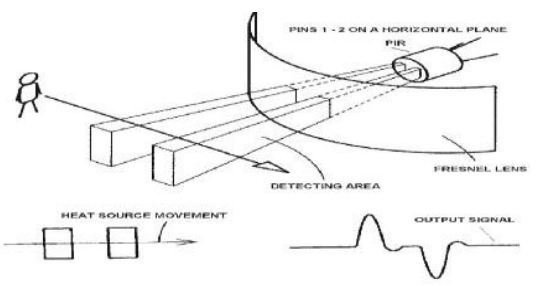

Fig. 4 (a). Heat Source Movement \& Detection Area

Table 2. 3 Bit Wide Gray Code

\begin{tabular}{|l|l|l|l|}
\hline S. No. & Bit 1 & Bit 2 & Bit 3 \\
\hline 1 & 0 & 0 & 0 \\
\hline 2 & 0 & 0 & 1 \\
\hline 3 & 0 & 1 & 0 \\
\hline 4 & 0 & 1 & 1 \\
\hline 5 & 1 & 0 & 0 \\
\hline 6 & 1 & 0 & 1 \\
\hline 7 & 1 & 1 & 0 \\
\hline
\end{tabular}

\section{SENSOR CHARACTERISTICS AND APPLICATIONS}

Passive infrared sensors are used to develop an electric signal in response to a change in the incident thermal radiation. Every living body emits some low level radiations and the hotter the body, the more is emitted radiation. Passive infrared system detects the change in the radiation of warm blood generation and completely used to turn On the webcam and lighting system [17].

They detect infrared light from some distance depending on how the device is calibrated.

PIR sensors are generally can be fitted into virtually any electronic device.

They do not need an external power source because they generate electricity as they absorb infrared light

The major applications areas of PIR Sensors are listed below:-

- All outdoor Lights

- $\quad$ Lift Lobby

- Multi Apartment Complexes

- Common staircases

- For Basement or Covered Parking Area

- $\quad$ Shopping Malls

- For garden lights 


\section{RESULTS}

The output of the sensor processing on it to emit a digital output pulse from the analog sensor is shown in the Fig 5.

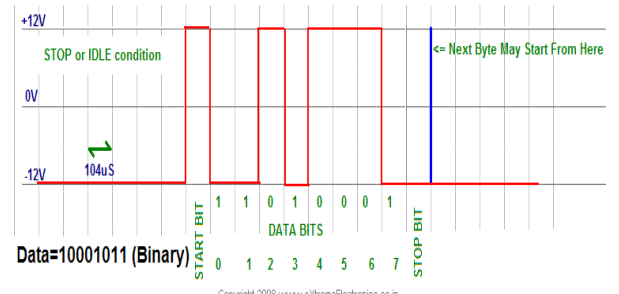

Fig. 5. Signal Generated by PIR

We can locate with simple information after ASK, PSK and FSK Modulation then encoding the perception areas of the sensors.

The system senses the signal generated by PIR sensor detecting the presence of individuals not at thermal equilibrium with the surrounding environment. Detecting the presence of any unauthorized person in any specific time interval, it triggers an alarm \& sets up a call to a predefined numbers are indicated in Fig.5. This highly reactive approach has low computational requirement, therefore it is well-suited to surveillance, industrial applications and smart environments. The low-power PIR detectors take advantage of pyro electricity to detect a human body that is a constant source of Passive Infrared (radiation in the infrared region) as shown in Fig 4. In addition PIR Sensor energy imbalance problem can be solving effectively with the PIR sensor detecting system [18].

\section{CONCLUSION}

This highly reactive approach has low computational requirement, therefore it is well-suited to surveillance, industrial applications and smart environments. The lowpower PIR detectors take advantage of pyro electricity to detect a human body that is a constant source of Passive Infrared.

In this security system PIR sensor has been used which is low power, and low cost, pretty rugged, have a wide lens range, and are easy to interface with. This security system can be implemented in places like home, office, shop etc. The sensitivity range for detecting motion of the system is about 3 to 4 feet. It can be raised up to 20 feet through careful use of concentrating optical lenses as future development. In addition to this, this system can be equipped with glass break detectors to enhance the level of protection. Use of multisensor data fusion and complex algorithm can be used to increase the effective FOV for larger spaces. In order to enhance the location accuracy and to enhance the method of processing the PIR sensor signal.

\section{REFERENCES}

[1] Liew, S. C. "Electromagnetic Waves". Centre for Remote Imaging, Sensing and Processing. Retrieved 2006-10-27.

[2] Jump up^ Sliney, David H.; Wangemann, Robert T.; Franks, James K.; Wolbarsht, Myron L. (1976). "Visual sensitivity of the eye to infrared laser radiation". Journal of the Optical Society of America 66 (4): 339341. doi:10.1364/JOSA.66.000339. (subscription required (help)).The foveal sensitivity to several nearinfrared laser wavelengths was measured. It was found that the eye could respond to radiation at wavelengths at least as far as $1064 \mathrm{~nm}$. A continuous $1064 \mathrm{~nm}$ laser source appeared red, but a $1060 \mathrm{~nm}$ pulsed laser source appeared green, which suggests the presence of second harmonic generation in the retina.

[3] Jump up^ Lynch, David K.; Livingston, William Charles (2001). Color and Light in Nature (2nd ed.). Cambridge, UK: Cambridge University Press. p. 231. ISBN 978-0521-77504-5. Retrieved 12 October 2013. Limits of the eye's overall range of sensitivity extends from about 310 to 1050 nanometers.

[4] Jump up^ Dash, Madhab Chandra; Dash, Satya Prakash (2009).Fundamentals Of Ecology 3E. Tata McGraw-Hill Education. $\quad$ p. 213.ISBN 978-1-259-08109-5 Retrieved 18 October 2013. Normally the human eye responds to light rays from 390 to $760 \mathrm{~nm}$. This can be extended to a range of 310 to $1,050 \mathrm{~nm}$ under artificial conditions.

[5] Jump up^ Saidman, Jean (15 May 1933). "Sur la visibilité de l'ultraviolet jusqu'à la longueur d'onde 3130" [The visibility of the ultraviolet to the wave length of 3130]. Comptes rendus de l'Académie des sciences(in French) 196: 1537-9.

[6] Jump up^ Michael Rowan-Robinson (2013). "Night Vision: Exploring the Infrared Universe". p. 23. Cambridge University Press,

[7] P.Turaga, R.Chellappa, V.S. Subrahmanian and O. Udrea, " Machine recognition of human activities : A Survey „IEEE Transactions on circuits and systems for video Technology, vol.18, No.11, pp.1473-1488,2008.

[8] Hernandez and M. Frean, "Bayesian multiple person tracking using probability hypothesis density smoothing „International Journal on smart sensing and Intelligent system, vol.4, No.2, pp.285-312,2011.

[9] L.Wang, W.Gong, L.He, H. Yiao and Y. Huang "Human motion recognition using pyro-electric infrared signal,, Journal of optoelectronics, laser, vol.21, No.3, pp.440-443,2010.

[10] W.Sen, C. Ying-Wen and X.ming, "Research of passive infrared wireless sensor network target tracking , Journal of transaction technology, vol.21, No.11, pp.1929-1934,2008.

[11] X.Zhao-Jun, Z.Peng-Pei and W.Bai-Kun,“ Application of infrared thermal imaging gait recognition, "Journal of optoelectronics , laser, vol.20, No.3, pp.402-405, 2010.

[12] R.Poppe," A survey on Vision based action recognition , " Image and Vision Computing, vol.28, No.6, pp.976990, 2010.

[13] Q.Hao, D.J . Brady, B.D.Guenther, J.B.Burchett, M.Shankar and S.Celler," Human Tracking with wireless distributed pyroelectric sensor, "IEEE Sensors Journal, vol.6, No.6, pp.1683-1695, 2006.

[14] .J.S.Fang, Q. Hao, D.J.Brady, B.D.Guenther and K.Y.Hsu," A pryelectric infrared biometric system for real time walker recognition by use of a maximum likelihood principal components estimation (MLPCE) method," Optics Express, vol.15, No.6, pp.3271-3284, 2007.

[15] .L.Suk, N.H.Kyang and C.L.Kyang,“ A pyroelectric infrared sensor based indoor location aware system for 
the smart home, "IEEE Transaction on consumer electronics , vol.52, No.4, pp.1311-1317, 2006.

[16] G.Xue-Bin, Z.Zhi-Qiang and Y.Shi-Wei,"Research of passive infrared sensor model for wireless sensor networks , "Journal of computer applications, vol.27, No.5, pp.1086-1089, 2007.
[17] Abhishek Pandey and R. C. Tripathi " A Survey on Wireless Sensor Networks", International Journal of Computer Applications, Vol 3, No. 2, pp 43-49, 2010.

[18] Nibedita Priyadarshini, Jagruti Jena and Sujata Kumari Sahu "Improving the Life of the Wireless Sensor Network using Energy Harvesting Clustering", International Journal of Computer Applications, Vol 114, No. 10, pp 37-43, March, 2015. 